\title{
Ferromagnetic state in the one-dimensional Kondo lattice model
}

\author{
Robert Peter:* and Norio Kawakami \\ Department of Physics, Kyoto University, Kyoto 606-8502, Japan
}

(Dated: September 25, 2018)

\begin{abstract}
In our recent study, Phys. Rev. Lett. 108086402 (2012), we have revealed the intriguing properties of the ferromagnetic state in the Kondo lattice model with antiferromagnetic coupling in infinite dimensions: within the ferromagnetic metallic phase, the minority conduction electrons form a gap at the Fermi energy and do not participate in transport irrespective of interaction strength and filling. This half-metallic state is referred to as a spin-selective Kondo insulator. We here show that the spin-selective Kondo insulator can also be realized in the one-dimensional Kondo lattice model by studying static and dynamical quantities with the density matrix renormalization group. The emergence of the spin selective Kondo insulator both in one and infinite dimensions certainly demonstrates that this mechanism is quite general and ubiquitous for the ferromagnetic state in the Kondo lattice model irrespective of the dimensionality of the system.
\end{abstract}

PACS numbers: 71.27.+a 75.10.-b 75.30.Mb

\section{INTRODUCTION}

The Kondo lattice model is one of the fundamental models for strongly correlated materials. It describes a lattice of local moments which are coupled locally to itinerant electrons. The Hamiltonian can be written as ${ }^{1-3}$

$$
H=t \sum_{<i, j>\sigma} c_{i \sigma}^{\dagger} c_{j \sigma}+J \sum_{i} \vec{S}_{i} \cdot \vec{s}_{i},
$$

where the first term describes electron hopping on a lattice and the second term the spin-spin interaction between a localized moment $\vec{S}$ and the spin of a conduction electron, $\vec{s}_{i}=c_{i \rho}^{\dagger} \vec{\sigma}_{\rho \rho^{\prime}} c_{i \rho^{\prime}}$ (with Pauli matrices $\vec{\sigma}$ ).

The Kondo lattice model is widely used for describing strongly correlated electron systems. Examples range from transition metal oxides such as the manganites $\underline{\underline{\underline{w}}}$ to heavy fermions. In the case of the manganites, the $d$ orbitals are split due to crystal fields into $e_{g^{-}}$and $t_{2 g^{-}}$ orbitals. Because of strong local interactions the halffilled $t_{2 g}$-orbitals, which lie energetically lower than the $e_{g}$-orbitals, can be approximated as localized spins, which are ferromagnetically coupled to the electrons in the $e_{g}$ band due to Hund's rule. Other examples are materials with partially filled f-orbitals, in which the electrons can be usually very well approximated as localized moments. However, a local hybridization between the $f$-orbitals and the itinerant electrons gives rise to an effective antiferromagnetic interaction between them at low temperatures. This second example describes systems such as heavy fermions and Kondo insulators. This article concentrates on the latter materials, which can be described by an antiferromagnetically coupled Kondo lattice model.

In our recent study, ${ }^{\frac{5}{5}}$ we have uncovered the intriguing properties of the ferromagnetic state in the Kondo lattice model (with antiferromagnetically coupled spins), which we call the "spin-selective Kondo insulator". It has been shown that while the majority conduction electrons are metallic, the minority conduction electrons form a gap at the Fermi energy, thus being insulating at low temperatures. The existence of such half-metallic states in the Kondo lattice model has been proposed in earlier works ${ }^{6-8}$ In our recent study we have demonstrated that the gap width follows a Kondo-type functional form, $T_{K} \sim \exp (-1 / J)$. Furthermore, we have clarified that the origin of this remarkable state is the cooperation between the localized spins and the conduction electrons. Namely, they adjust themselves in a way that the sum of the minority-spin conduction electrons and the localizedelectrons with the same spin always satisfies the condition of "one electron per lattice site". Remarkably, this commensurate situation is not only generated for a particular filling or combination of interaction parameters, but is ubiquitous for the ferromagnetic state in the antiferromagnetically coupled Kondo lattice model.

The above conclusion was drawn,,$\frac{5}{6}$ however, via the dynamical mean field theory (DMFT) $\underline{\underline{9}}$ The DMFT method, which maps the lattice model onto an impurity model embedded in a bath to be solved self-consistently, completely neglects spatial fluctuations, becoming exact in the limit of infinite dimensions. Therefore some serious questions naturally arise; whether the above conclusion depends on the method employed (infinite dimensions); what is really expected for low-dimensional systems, etc.

In this article, we address these questions by considering the opposite extreme, i.e. the one-dimensional (1D) Kondo lattice model, and demonstrate that the notion of the spin-selective Kondo insulator is fundamental for Kondo lattice systems. We explicitly show the emergence of the spin selective Kondo insulator in the ferromagnetic phase of the 1D lattice model by using the density matrix renormalization group (DMRG),$^{10-12}$ which provides an excellent tool to calculate the ground state quantities in $1 \mathrm{D}$ with very high precision. An important point is that DMRG can precisely take into account spatial fluctuations that were neglected in the DMFT study.

The paper is organized as follows. After revisiting the ground state phase diagram in 1D briefly in Sec.II, we analyze static as well as dynamical quantities of the 1D Kondo lattice model in Sec. III. We also compare the present results in $1 \mathrm{D}$ with those obtained by DMFT, and 


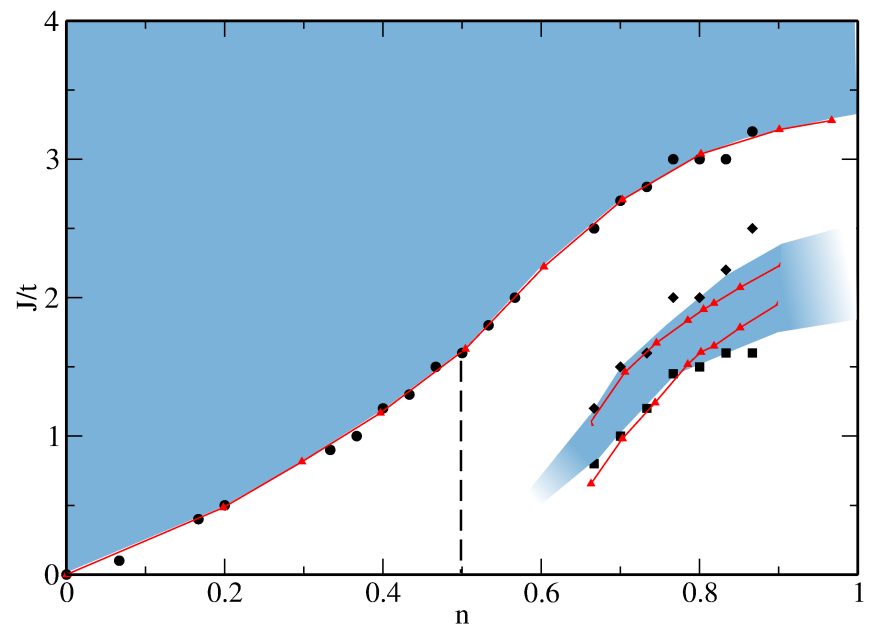

Figure 1: (Color online) Phase diagram of the 1D Kondo lattice model. Black symbols represent the phase boundaries which are obtained in this work. The calculations are performed using $L=60$ - and $L=120$-site chains and $m=800-1200$ states for the density matrix. Only Abelian symmetries are taken into account. Red lines and symbols are taken from McCulloch et al. $\stackrel{16}{ }$ Blue (white) color represents the ferromagnetic (paramagnetic) phase.

show that the ferromagnetic state possesses very similar properties for both cases. Through these comprehensive analyses in $1 \mathrm{D}$, together with the previous DMFT results in infinite dimensions, we demonstrate that the spin-selective Kondo insulator is general and fundamental for the ferromagnetic state of the Kondo lattice model in any dimension. Sec. IV is devoted to a brief summary of the paper.

\section{PHASE DIAGRAM AT ZERO TEMPERATURE REVISITED}

Let us start with an overview of the magnetic phase diagram of the 1D Kondo lattice model away from half filling. We assume that the coupling between localized spins and conduction electrons is antiferromagnetic. The phase diagram for the 1D Kondo lattice model has been calculated by a number of groups before $\underline{\underline{13}} \underline{-18}$ In order to clarify the parameter regime where the ferromagnetism is stabilized, we show the phase diagram in Fig. 1] We compare our phase boundaries to those of McCulloch et al. $\stackrel{16}{n}$ obtained by using non-Abelian DMRG with $\mathrm{SU}(2)$ symmetry (note that our results resort to only Abelian symmetries). The phase diagram includes two ferromagnetic phases away from half filling. The first ferromagnetic phase extends from weak coupling and low filling of the conduction electrons. For strong coupling, this ferromagnetic phase extends in the whole metallic region away from half filling. For intermediate coupling within the paramagnetic phase, there is another ferromagnetic phase, which was firstly identified by using non-

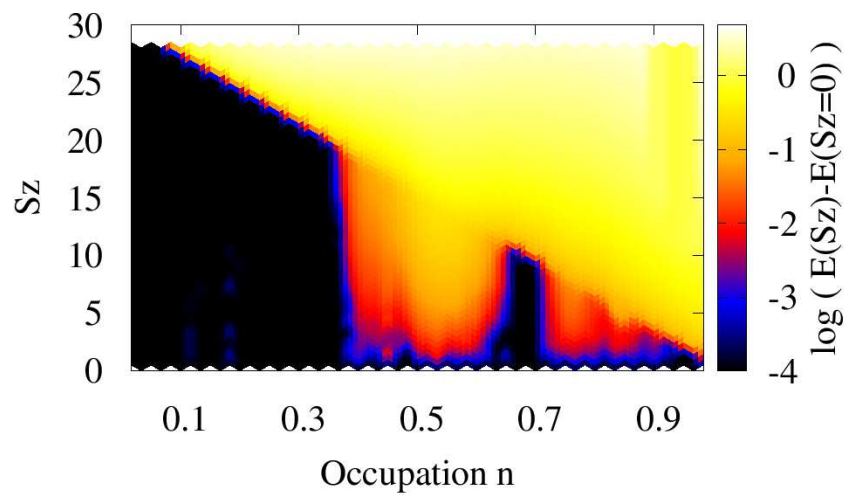

Figure 2: (Color online) Energy differences on a logarithmic scale for the 1D Kondo lattice model calculated with DMRG for $J=t$ and $m=800$ states kept within DMRG. For each filling the energy for $S_{z}^{\text {tot }}=0\left(E\left(S_{z}^{\text {tot }}=0\right)\right)$ is subtracted. In this way the ferromagnetic phases can be clearly identified without using $\mathrm{SU}(2)$-symmetry-conserving calculations.

Abelian DMRG $\underline{16}$ It was stated that this phase (intermediate coupling strengths and fillings $n_{c}>0.5$ ) is very difficult to observe in the DMRG calculations conserving only Abelian symmetries $\stackrel{16}{\underline{1}}$ In the present study, however, we can clearly observe this ferromagnetic phase at intermediate coupling strengths.

Our strategy is as follows. To identify the ferromagnetic phases we first determine the energetically lowest eigenstate of all $S_{z}^{\text {tot }}$-sectors, and calculate the energy difference between different $S_{z}^{\text {tot }}$-sectors, $E\left(S_{z}^{\text {tot }} \neq\right.$ $0)-E\left(S_{z}^{t o t}=0\right)$. For a ferromagnetic state, all states fulfilling $S_{z}^{\text {tot }} \leq \mathcal{S}^{\text {tot }}\left(\mathcal{S}^{\text {tot }}\right.$ is the $\mathrm{SU}(2)$ spin quantum number of the ground state) are supposed to be energetically degenerate. Plotting these energy differences for different fillings on a logarithmic scale, one can clearly identify two distinct ferromagnetic phases, where the energies do not change for $S_{z}^{\text {tot }} \leq \mathcal{S}^{\text {tot }}$. An example for this procedure for $J=t$ is shown in Fig. 2,

In this way, we can deduce the phase diagram of the 1D Kondo lattice model containing two ferromagnetic phases (blue area in Fig. 11). Having found good agreement in the phase boundaries of the ferromagnetic phases with those obtained by the $\mathrm{SU}(2)$ calculations, $\stackrel{16}{\longleftarrow}$ we believe that our calculations are accurate enough to analyze the detailed structure of the ferromagnetic states.

\section{FERROMAGNETIC STATE AT LOW FILLINGS}

\section{A. static quantities}

In the following we focus on the ferromagnetic state in the 1D Kondo lattice model. In the strongly coupled Kondo lattice with $J \rightarrow \infty$, all electrons are supposed to be bound in singlet states. Nevertheless, in order to create a ferromagnetic state, a fraction of the electrons 
should not be bound in singlets. These electrons "connect" the different lattice sites and stabilize the magnetic state. To clarify the internal structure of the spinselective Kondo insulator, we firstly look at static quantities.

As mentioned above, the ferromagnetic state in infinite dimensions has some intriguing properties,$\stackrel{5}{,}$ the oneparticle spectral function of the minority electrons shows a gap at the Fermi energy. The mechanism of this gap formation is the commensurability arising from the cooperation between localized- and conduction-electrons; they arrange themselves so that

$$
\left\langle n_{\downarrow, i}^{f}\right\rangle+\left\langle n_{\downarrow, i}^{c}\right\rangle=1
$$

holds for any lattice site $i$. Here we have assumed that the localized moment is formed by a half filled $f$-orbital and the spin polarization is written as $\left\langle S_{z, i}\right\rangle=\frac{1}{2}\left(\left\langle n_{\uparrow, i}^{f}\right\rangle-\right.$ $\left.\left\langle n_{\downarrow, i}^{f}\right\rangle\right)$, which is the case for heavy fermion materials.

The essence of the commensurability may be understood clearly via the following arguments. Imagine first the Kondo lattice model at half filling, which shows the Kondo-gap formation under the commensurability condition: the sum of the averaged number of conduction and localized electrons at each site is unity per spin direction. This is the well-known mechanism of generating the Kondo insulator. For a system away from half filling, a correlated metallic phase is stabilized. However, if the system is very close to half filling, small magnetic fields can induce a spin polarization and simultaneously drive the system into a half-metallic (majority-spin conduction electrons) and half-insulating (minority-spin conduction electrons) state, where only the electrons in the insulating sector satisfies the commensurability (half filling) condition. This exemplifies the commensurability away from half filling in finite magnetic fields. What is nontrivial and remarkable for the spin-selective Kondo insulator is that the commensurability is generated concomitantly with the ferromagnetic transition. In particular, it emerges for a metallic system far away from half filling, where there is no sign of the gap-formation in the normal metallic phase. Even in such low-filling cases, once the ferromagnetism is spontaneously induced by the Kondo exchange interaction, the emergent commensurability plays an essential role in generating a finite gap at the Fermi level, which in turn stabilizes the ferromagnetic state energetically.

For the 1D Kondo lattice model it has been known from the studies for the one-electron case $\frac{19}{}$ and the exact diagonalization calculations 20 that the quantum number of the ferromagnetic ground state for strong enough coupling strength $J$ is

$$
\mathcal{S}^{t o t}=1 / 2|L-N|,
$$

where $\mathcal{S}^{\text {tot }}$ denotes the total $\mathrm{SU}(2)$-spin quantum number, $L$ the number of lattice sites, and $N$ the number of conduction electrons. In a classical picture, a state with this spin quantum number describes the situation in which all localized spins are polarized in one direction and all electrons point to the opposite direction. However, this is not the case for our model where the Kondo effect does work, reducing the polarization of conduction as well as localized electrons via large quantum fluctuations.

We now show that the ground state having a spin quantum number $\mathcal{S}^{\text {tot }}=1 / 2|L-N|$ in $1 \mathrm{D}$ is a direct consequence of the emergent commensurability, which was introduced in the DMFT calculations $\frac{\sqrt{5}}{\text { Suppose that }}$ the symmetry is spontaneously broken and the maximal $S_{z}^{\text {tot }}$ value represents the homogeneous ground state for a given $\mathrm{SU}(2)$ spin quantum number $\mathcal{S}^{\text {tot }}$. Then we can derive the commensurability by just inserting the definitions of the local quantities:

$$
\begin{aligned}
S_{z}^{\text {tot }} & =1 / 2\left(N_{c}-N\right) \\
\left(N_{\uparrow}^{f}-N_{\downarrow}^{f}\right)+\left(N_{\uparrow}^{c}-N_{\downarrow}^{c}\right) & =N^{c}-N \\
\left(n_{\uparrow}^{f}-n_{\downarrow}^{f}\right)+\left(n_{\uparrow}^{c}-n_{\downarrow}^{c}\right) & =n_{\uparrow}^{c}+n_{\downarrow}^{c}-1 \\
\left(n_{\uparrow}^{f}-n_{\downarrow}^{f}\right) & =2 n_{\downarrow}^{c}-1 \\
2\left(n_{\downarrow}^{c}+n_{\downarrow}^{f}\right) & =2 .
\end{aligned}
$$

In the second line, the local expectation value is calculated by averaging over all lattice sites (corresponding to a homogeneous state). Furthermore a half-filled f-orbital is assumed.

We thus come to the conclusion: the fact that the ferromagnetic ground state in the $1 \mathrm{D}$ Kondo lattice model is associated with the spin quantum number $\mathcal{S}^{\text {tot }}=1 / 2(L-$ $N$ ) is equivalent to the emergent commensurability in our DMFT calculations. $\frac{5}{2}$ However, if one chooses a different state, $\left|S_{z}^{\text {tot }}\right|<\mathcal{S}^{\text {tot }}$, as the ground state, the commensurability is not easily visible, because the spin channels are mixed. Therefore, if the commensurability condition is satisfied, the highest $S_{z}^{\text {tot }}$-value of the energetically degenerate states in Fig. 22 for which $\left|S_{z}^{t o t}\right|=\mathcal{S}^{\text {tot }}$ holds, should feature a straight line, $S_{z}^{\text {tot }}=1 / 2(L-N)$. One can see that this is indeed the case in Fig. 2, Thus, the emergent commensurability allows us to use the relation $\mathcal{S}^{\text {tot }}=1 / 2(L-N)$ not only for the strong coupling regime as originally suggested, $\stackrel{19}{\longleftarrow}$ but also for the weak coupling regime.

We have performed calculations for a finite-size Kondo lattice model. The introduction of open boundaries induces oscillations in all measured quantities. In Fig. 3 we show how the local commensurability behaves depending on the lattice site in the ferromagnetic state with $S_{z}^{\text {tot }}=1 / 2(L-N)$, for an $L=60$-sites 1D Kondo lattice model. Although the commensurability must be fulfilled when averaged over all sites, it is not necessarily fulfilled locally in a finite size system with open boundaries. In Fig. 3 we can see that, for the ferromagnetic states at strong coupling (upper 4 panels), the commensurability is almost fulfilled locally within one percent, where small oscillations are due to open boundaries. On the other hand, the "second" ferromagnetic phase (lower panel) at intermediate coupling strengths shows very strong oscil- 


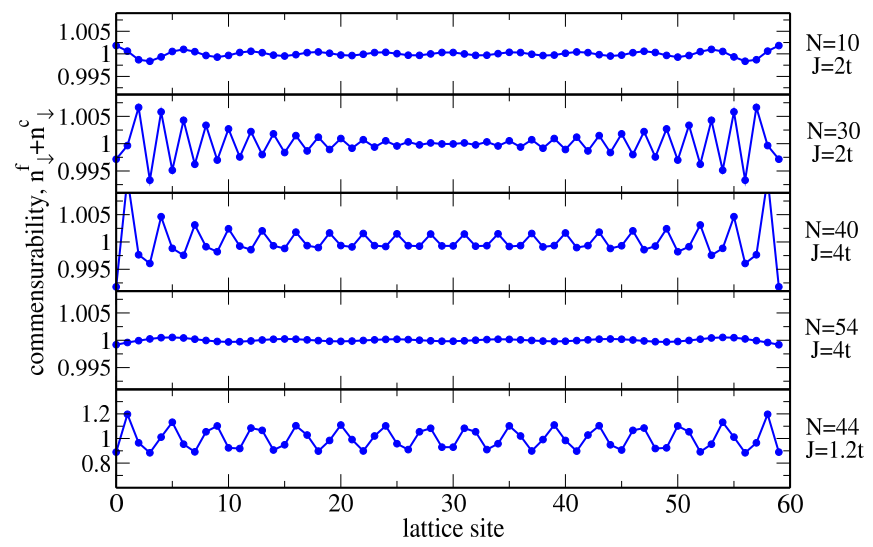

Figure 3: (Color online) Locally measured commensurability, $\left\langle n_{\downarrow, i}^{c}+n_{\downarrow, i}^{f}\right\rangle=\left\langle n_{\downarrow, i}^{c}\right\rangle-\left\langle S_{z, i}\right\rangle+0.5$, in the finite size 1D Kondo lattice model. Calculations were performed for a 60 sites chain using $m=800$ states. While the upper four panels show examples of the strong coupling ferromagnet, the lowest panel shows the "second" ferromagnetic phase for intermediate couplings. The interaction parameters are given on the right side of each panel. Lines are guide to eye.

lations. The overall tendency implies that oscillations are stronger near quarter filling and weak coupling.

We present a schematic picture in Fig. [4 which may help to imagine how the ferromagnetic state looks under the emergent commensurability. Given that the localized spin is formed by a half-filled strongly correlated f-electron, then the commensurability condition leads to the situation in which the number of minority conduction electrons (spin up in Fig. (4) is equal to that of minority f-electrons (spin down in Fig. 44). Thus, the total number of spin up electrons becomes unity. In this situation, the minority conduction electrons collaborate with the minority localized electrons, together with the same fraction of opposite majority electrons, to form a Kondo singlet state, while the rest of the majority conduction electrons and localized spins form a ferromagnetic state.

A close examination of local expectation values enables us to analyze the ferromagnetic state in more detail. In Fig. 5 and Fig. 6 we compare static expectation values for different fillings and different coupling strengths. The expectation values are calculated for finite-size chains and averaged over all lattice sites. The commensurability is fulfilled for all shown data points. Thus, the expectation value of the localized spin $\left\langle S_{z}\right\rangle$ is directly related to the occupation number of the minority conduction electrons. The expectation value $\left\langle S_{z} s_{z}\right\rangle$, which represents the local spin-spin correlation between conduction electron and localized spin, fulfills the condition $\left\langle S_{z} s_{z}\right\rangle>-0.25\left\langle n_{\uparrow}+n_{\downarrow}\right\rangle$. If all conduction electrons would be bound in local singlets this spin-spin correlation is supposed to be equal to one quarter of the occupation. If we associate these expectation values with the state shown in Fig. 4. then the polarization, $\left\langle n_{\uparrow}-n_{\downarrow}\right\rangle$, corresponds to the number of majority electrons that are

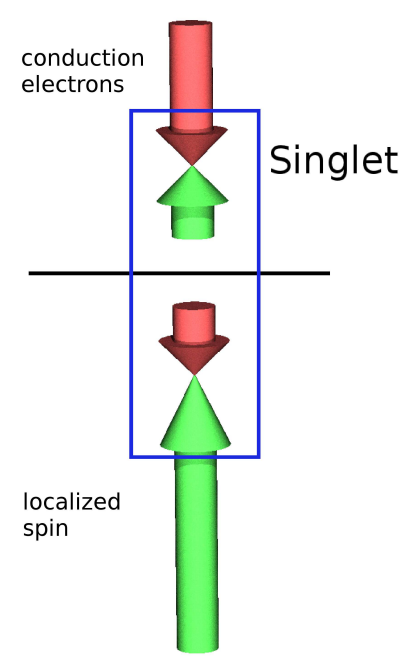

Figure 4: (Color online) Sketch of a state fulfilling the commensurability. The upper (lower) part corresponds to the conduction electrons (localized spins). The commensurability is equivalent to the fact that there are the same number of minority conduction electrons (here: spin up) and minority f-electrons (here spin down).

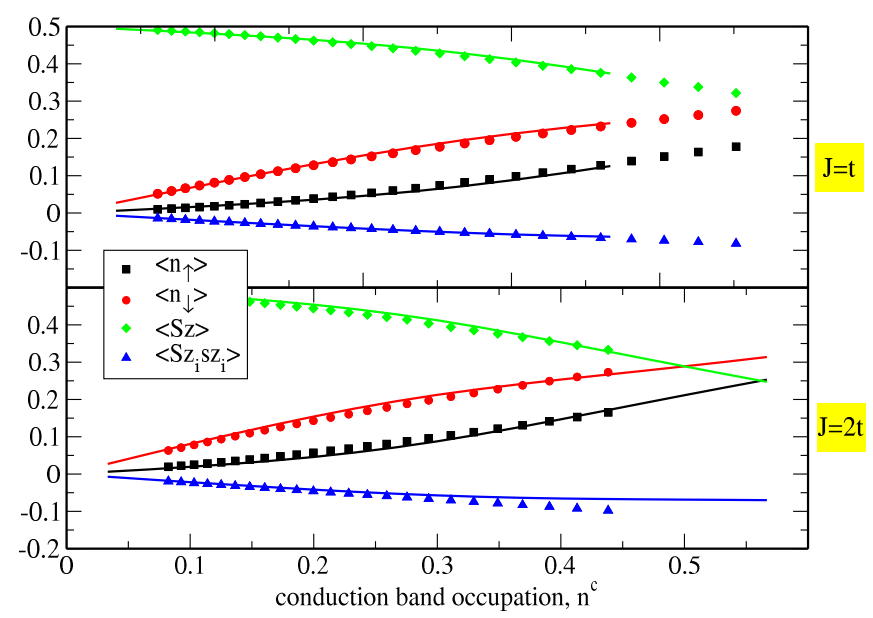

Figure 5: (Color online) Local expectation values for different lattice fillings, averaged local occupation $n^{c}=\left\langle n_{\uparrow}^{c}\right\rangle+\left\langle n_{\downarrow}^{c}\right\rangle$ (1D: $n_{c}=N / L \mathrm{~N}$ : electron number L: length of the chain), in the ferromagnetic state with $S_{z}^{\text {tot }}=1 / 2(L-N)$. Continuous lines correspond to the 1D Kondo lattice model calculated for $L=60$-sites chains and averaged over all lattice sites. For comparison we include the results of the previous DMFT calculations which are plotted as four different symbols (coupling strengths: upper panels $J=t$, lower panels $J=2 t$ ).

not bound in local singlets. Therefore, for interaction strengths $|J| \approx|t|$ the number of electrons bound in local singlets and the number of polarized electrons are roughly equal.

In Fig. 5 we also include the expectation values obtained by DMFT calculations,,$\frac{5}{5}$ and find that the values of DMFT and DMRG are very close to each other. This 

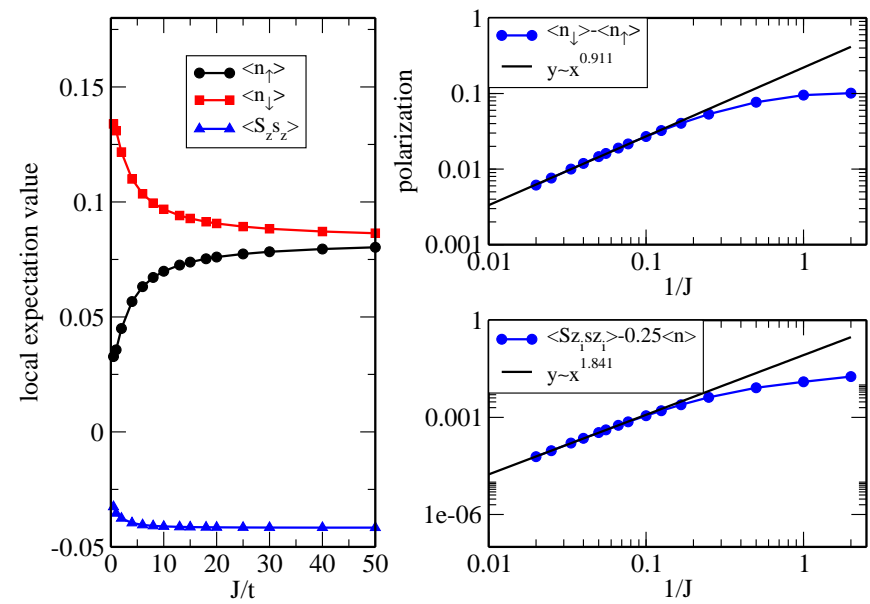

Figure 6: (Color online) Local expectation values for the 1D Kondo lattice model, calculated for $L=60$ sites chain and $N=10$ electrons, thus $n=N / L \approx 0.17$ ( $m=800$ states kept in DMRG). Right upper panel shows a power-law-fitting to the electron magnetization. The right lower panel shows a power-law-fitting to the spin-spin correlation function which approaches $-1 / 4\left\langle n_{\uparrow}+n_{\downarrow}\right\rangle$ in the strong coupling limit. (Lines are guide to the eye.)

is remarkable because the phase boundaries calculated by DMFT show completely different behavior from the present 1D case. Therefore, we can say that local expectation values of the ground state are not sensitive to the dimensionality, and are controlled mainly by electron filling and interaction strength. On the other hand, the transition point between the paramagnet and ferromagnet strongly depends on the lattice geometry. This suggests that the contribution of the kinetic energy plays a key role in determining which has the lowest energy, the ferromagnetic state or the paramagnetic state.

Let us now discuss how the ferromagnetic state behaves when increasing the interaction strength from weak to strong coupling. In the strong coupling limit, the ferromagnetic phase in the 1D Kondo lattice model covers the whole phase diagram except half filling where the system is paramagnetic. In Fig. 6 we compare local expectation values in the $1 \mathrm{D}$ lattice for different coupling strengths. The filling is fixed at $\langle n\rangle \approx 0.17$. It is seen that the electron polarization is larger for weak coupling. With increasing coupling strength the polarization of the electrons decreases and vanishes as a power law, as shown in the upper right panel of Fig. 6. The exponent depends on the electron filling. In a similar way, the expectation value for the localized spin polarization (not shown), $\left\langle S_{z}\right\rangle$, approaches a finite value, $\left\langle S_{z}\right\rangle=0.5-\left\langle n_{\uparrow}\right\rangle$, given by the commensurability. Also the local spin-spin correlation $\left\langle S_{z} s_{z}\right\rangle$ between the conduction electron and the spin approaches a fixed value, given by $\left\langle S_{z} s_{z}\right\rangle \rightarrow-0.25\left(\left\langle n_{\uparrow}\right\rangle+\left\langle n_{\downarrow}\right\rangle\right)$. All these quantities show power-law behavior in the strong coupling regime. The monotonic power-law decrease in the electron polarization, as well as $\left\langle S_{z} s_{z}\right\rangle \rightarrow-0.25\left(\left\langle n_{\uparrow}\right\rangle+\left\langle n_{\downarrow}\right\rangle\right)$, implies that most of electrons are bound into local singlets for large $J$. In the limit of infinitely strong coupling $J$ where $\left\langle n_{\downarrow}\right\rangle=\left\langle n_{\uparrow}\right\rangle$ and $\left\langle S_{z} s_{z}\right\rangle=-0.25\left(\left\langle n_{\uparrow}\right\rangle+\left\langle n_{\downarrow}\right\rangle\right)$ all the conduction electrons are bound in local singlets.

\section{B. Spectral properties}

Having studied the static quantities, let us now look into spectral functions with particular focus on the gap created for the minority conduction electrons. We have so far clarified that the mechanism of the gap formation in the ferromagnetic state is the emergent commensurability for the minority conduction electrons. A glance at the energy values presented in Fig. 2 clearly shows that there is a jump in the energy between the ferromagnetic state and the next excited state. Assuming that the ground state of the system is the state with quantum number $S_{z}^{\text {tot }}=1 / 2(L-N)$ (in the ferromagnetic phase), which is realized if an arbitrarily small magnetic field is applied in the $z$-direction, then the single particle spectral function for the conduction electrons is given by excitations between the ferromagnetic ground state and excited states with a quantum number $S_{z}^{t o t}=1 / 2(L-N)+1$. In Fig. 2. there is an energy gap between these two states. Furthermore, we can see that the second ferromagnetic phase at intermediate couplings also fulfills the commensurability, and that there is an energy gap between the ground state and these excited states.

We show in Fig. 7 the local spectral functions of conduction electrons in both ferromagnetic phases. The state at $(J / t=1,\langle n\rangle=0.25)$ corresponds to the ferromagnetic state extending from low fillings, while the state at $(J / t=1.8,\langle n\rangle=0.8)$ corresponds to the second ferromagnetic phase at intermediate coupling strengths. For the calculation we have used the state corresponding to $S_{z}^{t o t}=1 / 2(L-N)$. We have performed a correction-vector calculation 21,22 with Lorentzian broadening $\eta / t=0.05$. Because of this broadening the spectral functions of the minority electrons (upper panels) show only a dip but no real gap at the Fermi energy. To improve the resolution we have calculated a delta-peak excitation spectrum corresponding to a deconvoluted spectral function. ${ }^{23}$ It is now seen from the computed excitation spectrum that the spectral functions of the minority conduction electrons indeed have a gap at the Fermi energy for both ferromagnetic phases in the 1D Kondo lattice model. Note that the ferromagnetic state at intermediate coupling strengths also has a gap for the majority conduction electrons, but slightly above the Fermi energy. This is the expected behavior, as both spin channels should be gapped for the half-filled lattice. Although the mechanism for the formation of two ferromagnetic phases might be different, the ground state shows very similar properties fulfilling the commensurability and having a gap for the minority conduction electrons.

In Fig. 8, we show the momentum resolved spectral function for $(J / t=1,\langle n\rangle=0.25)$. The shape of the spec- 

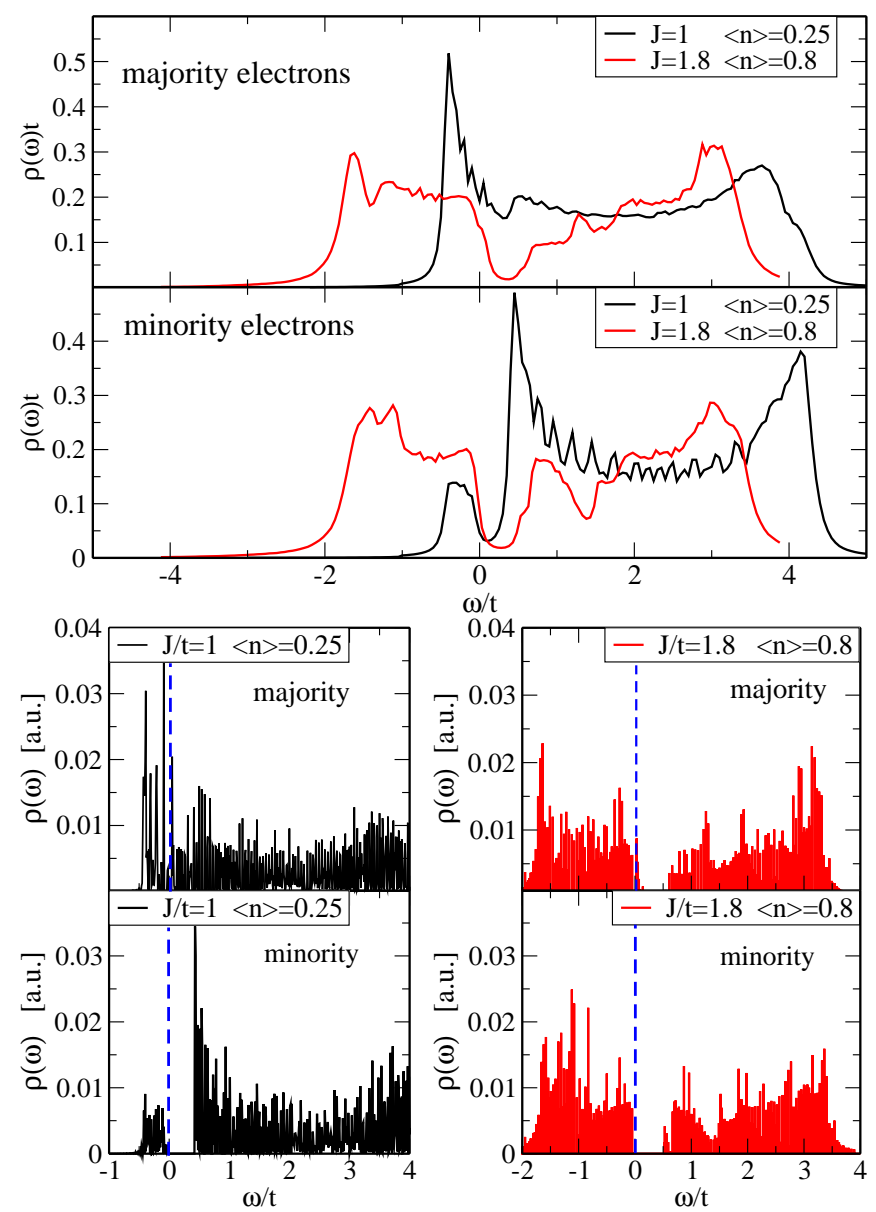

Figure 7: (Color online) Local spectral functions for the ferromagnetic state with $S_{z}=1 / 2(L-N)$ in the $1 \mathrm{D}$ Kondo lattice model. The spectral functions are calculated by the correction vector method for an $L=40$-sites chain using $m=400$ states and $\eta / t=0.05$. The Fermi energy lies at $\omega=0$. Upper panels: Local spectral functions for $(J / t=1$, $\langle n\rangle=0.25)$ corresponding to a ferromagnetic state at low filling, and for $(J / t=1.8,\langle n\rangle=0.8)$ corresponding to a state in the second ferromagnetic phase). Lower panel: Deconvoluted spectral functions (see text), which describe the excitations in the spectral function. The blue line corresponds to the Fermi energy.

trum looks very similar for both spin directions. However, while the lower band for the minority conduction electrons terminates below the Fermi energy, the lower band for the majority conduction electrons crosses the Fermi energy. To see the gap in the spectrum, it is important to choose $S_{z}^{\text {tot }}= \pm 1 / 2(L-N)$ as a quantum number for the ground state. Note that in the previous DMRG calculations with $\mathrm{SU}(2)$ symmetry, $\stackrel{24}{\rightleftharpoons}$ the gap at the Fermi energy is not visible, because both spin channels are mixed.

Finally, in Fig. 9 we compare the gap width for different interaction strengths. We also include the results from our DMFT calculations. $\underline{\underline{5}}$ It is seen that the gap in the DMFT results nicely follows a Kondo-type func-
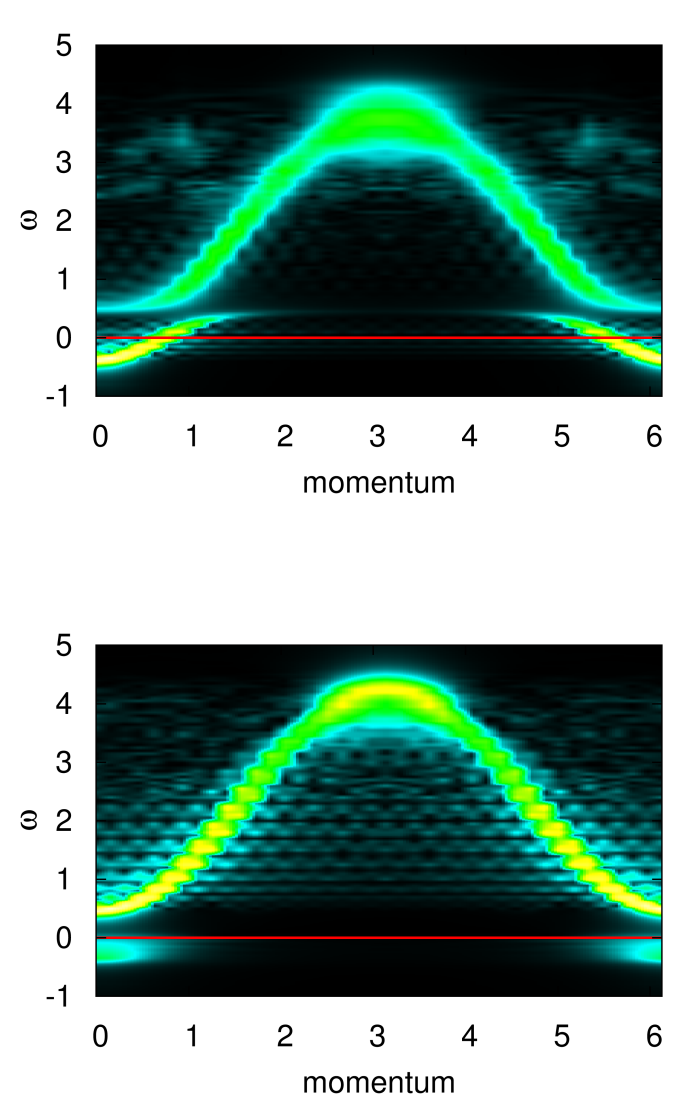

Figure 8: (Color online) Momentum-resolved spectral functions for the 1D Kondo lattice model in the ferromagnetic state. The red line represents the Fermi energy. The upper and lower panel show the spectral functions for the majorityand minority-conduction electrons, respectively. The interaction parameter and filling are $J=t$ and $\langle n\rangle=0.25$. The spectral functions are calculated by the correction vector method for an $L=40$-sites chain using $m=400$ states and $\eta / t=0.05$.

tional form $T_{K} \sim \exp (-1 / J)$. The curves for the $1 \mathrm{D}$ Kondo lattice model shown in Fig. 9, which may not follow an exponential-type behavior, terminate at the phase boundary to the paramagnetic solution at weak coupling. Generally, the gap width of the 1D Kondo lattice model is larger than in the DMFT results. Furthermore, the gap width in the 1D lattice is linear in the coupling strength for strong coupling, showing derivations in the vicinity of the phase boundary. As these derivations occur only in a very small region around the phase boundary, it is very hard to tell the functional form of the gap width in this region. However, the deviation from an exponential form can be understood from the known fact that the Kondo temperature in a 1D Tomonaga-Luttinger liquid takes a power-law form depending on the system parameters $\underline{25}$ We therefore believe that the gap width corresponds to the energy scale of the Kondo temperature in a $1 \mathrm{D}$ lat- 


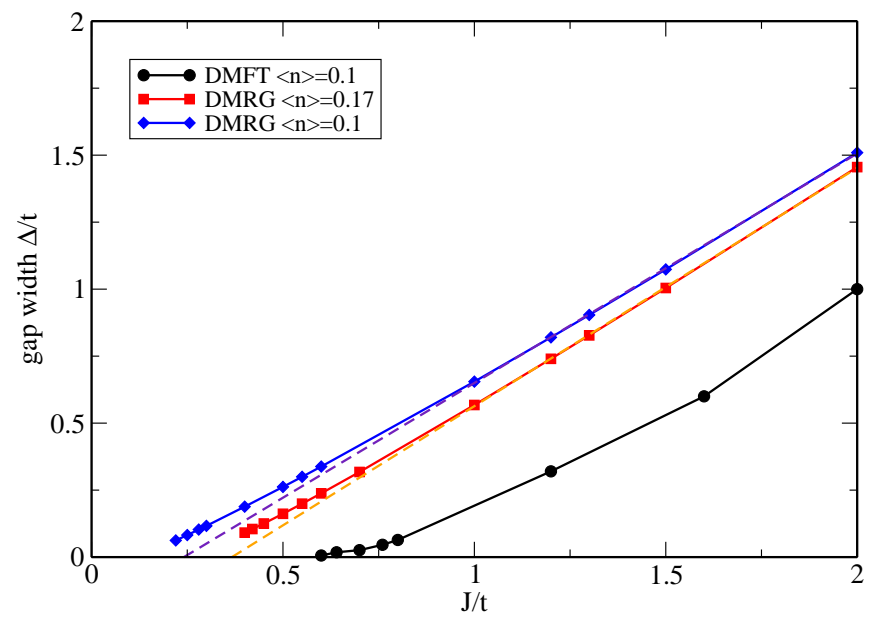

Figure 9: (Color online) Comparison of the gap width between the ferromagnetic state in infinite dimensions (DMFT results) and one dimension (DMRG results). The dashed lines represent linear fits to the strong coupling region.

tice.

\section{SUMMARY}

We have studied the ferromagnetic state in the 1D Kondo lattice model. Using the density matrix renormalization group, we have calculated the phase diagram and confirmed the existence of two ferromagnetic phases. Motivated by our recent findings based on DMFT in infinite dimensions, we have focused on the spectral func- tions in the ferromagnetic state. It has been elucidated that, similar to DMFT, the spin selective Kondo insulator is indeed realized in the $1 \mathrm{D}$ lattice, in which the minority conduction electrons form a Kondo gap at the Fermi energy, whereas the majority conduction electrons stay metallic. This gap is stabilized by the emergent commensurability: the number of minority conduction electrons, together with the localized electrons with the same spin, should be unity in the ferromagnetic phase. It has also been shown that the commensurability is equivalent to the statement that the ground state in the ferromagnetic phase is given by a state with spin quantum number $\mathcal{S}^{\text {tot }}=1 / 2(L-N)$. The commensurability and the corresponding gap formation have indeed been observed in the two distinct ferromagnetic phases of the 1D Kondo lattice model. Through these comprehensive analyses in one and infinite dimensions, we have confirmed that the spin-selective Kondo insulator is the fundamental notion for the ferromagnetic state of the Kondo lattice model in any dimension.

\section{Acknowledgments}

We acknowledge fruitful discussions with A. Koga and Y. Tada, T. Pruschke, G. Khaliullin, S. Hoshino, Y. Kuramoto, and N. Shibata. RP thanks the Japan Society for the Promotion of Science (JSPS) and the Alexander von Humboldt-Foundation for support during a 2 years fellowship and for the following support from JSPS by its FIRST Program. NK is supported by KAKENHI (No. 20102008) and JSPS through its FIRST Program. The calculations were performed at the ISSP in Tokyo.

* peters@scphys.kyoto-u.ac.jp

1 S. Doniach, Physica B 91, 231 (1977).

2 C. Lacroix and M. Cyrot, Phys. Rev. B 20, 1969 (1979).

3 P. Fazekas and E. Muller-Hartmann, Z. Phys. B: Condens. Matter 85, 285 (1991).

4 E. Dagotto, T. Hotta, and A. Moreo, Physics Reports 344, 1 (2001).

${ }^{5}$ R. Peters, N. Kawakami, and T. Pruschke, Phys. Rev. Lett. 108, 086402 (2012).

${ }^{6}$ V. Y. Irkhin and M. I. Katsnelson, Zeitschrift für Physik B Condensed Matter 82, 77 (1991).

7 K. S. D. Beach and F. F. Assaad, Phys. Rev. B 77, 205123 (2008).

8 S. Viola Kusminskiy, K. S. D. Beach, A. H. Castro Neto, and D. K. Campbell, Phys. Rev. B 77, 094419 (2008).

9 A. Georges, G. Kotliar, W. Krauth, and M. Rozenberg, Rev. Mod. Phys. 68, 13 (1996).

10 S. White, Phys. Rev. Lett. 69, 2863 (1992).

11 U. Schollwöck, Rev. Mod. Phys. 77, 259 (2005).

12 U. Schollwöck, Annals of Physics 326, 96 (2011).

13 M. Troyer and D. Würtz, Phys. Rev. B 47, 2886 (1993).

14 H. Tsunetsugu, M. Sigrist, and K. Ueda, Rev. Mod. Phys. 69, 809 (1997).

15 I. McCulloch, A. Juozapavicius, A. Rosengren, and M. Gulácsi, Philosophical Magazine Letters 81, 869 (2001).

16 I. P. McCulloch, A. Juozapavicius, A. Rosengren, and M. Gulacsi, Phys. Rev. B 65, 052410 (2002).

17 M. Gulácsi *, Advances in Physics 53, 769 (2004).

18 S. A. Basylko, P. H. Lundow, and A. Rosengren, Phys. Rev. B 77, 073103 (2008).

19 M. Sigrist, H. Tsunetsuga, and K. Ueda, Phys. Rev. Lett. 67, 2211 (1991).

${ }^{20}$ H. Tsunetsugu, M. Sigrist, and K. Ueda, Phys. Rev. B 47, 8345 (1993).

21 T. Kühner and S. White, Phys. Rev. B 60, 335 (1999).

22 E. Jeckelmann, Phys. Rev. B 66, 045114 (2002).

23 R. Peters, Phys. Rev. B 84, 075139 (2011).

24 S. Smerat, U. Schollwöck, I. P. McCulloch, and H. Schoeller, Phys. Rev. B 79, 235107 (2009).

25 A. Furusaki and N. Nagaosa, Phys. Rev. Lett. 72, 892 (1994). 